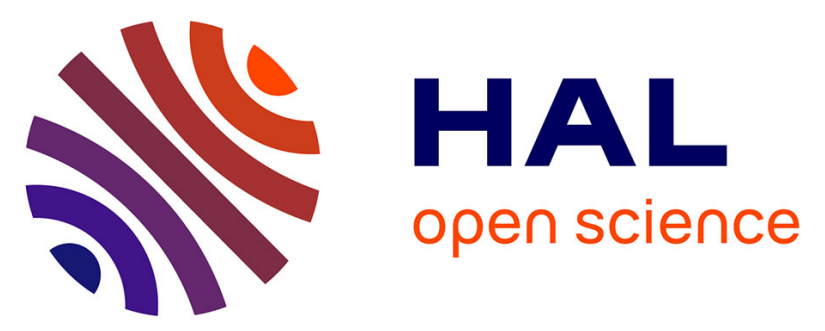

\title{
Adverse Effects of Oseltamivir Phosphate Therapy on the Liver of LDLR 2/2 Mice Without Any Benefit on Atherosclerosis and Thrombosis
}

Olivier Bocquet, Amandine Wahart, Thomas Sarazin, Elise Vincent, Christophe Schneider, Anne Fougerat, Stéphanie Gayral, Aubéri Henry, Sébastien Blaise, Béatrice Romier-Crouzet, et al.

\section{To cite this version:}

Olivier Bocquet, Amandine Wahart, Thomas Sarazin, Elise Vincent, Christophe Schneider, et al.. Adverse Effects of Oseltamivir Phosphate Therapy on the Liver of LDLR $2 / 2$ Mice Without Any Benefit on Atherosclerosis and Thrombosis. Journal of Cardiovascular Pharmacology, 2021, 77 (5), pp.660-672. 10.1097/FJC.0000000000001002 . hal-03358077

\section{HAL Id: hal-03358077 https://hal.science/hal-03358077}

Submitted on 1 Oct 2021

HAL is a multi-disciplinary open access archive for the deposit and dissemination of scientific research documents, whether they are published or not. The documents may come from teaching and research institutions in France or abroad, or from public or private research centers.
L'archive ouverte pluridisciplinaire HAL, est destinée au dépôt et à la diffusion de documents scientifiques de niveau recherche, publiés ou non, émanant des établissements d'enseignement et de recherche français ou étrangers, des laboratoires publics ou privés. 


\section{Adverse effects of oseltamivir phosphate therapy on the liver of $\operatorname{LDLR}^{-/-}$} mice without any benefit on atherosclerosis and thrombosis

Olivier Bocquet $^{1}$, Dr. Amandine Wahart ${ }^{1}$, Thomas Sarazin ${ }^{1}$, Elise Vincent ${ }^{1}$, Dr. Christophe

5 Schneider $^{1}$, Dr. Anne Fougerat ${ }^{2}$, Dr. Stéphanie Gayral ${ }^{2}$, Aubéri Henry ${ }^{1}$, Dr. Sébastien Blaise ${ }^{1}$,

Dr. Béatrice Romier-Crouzet ${ }^{1}$, Dr. Stéphane Jaisson ${ }^{1,3}$, Pr. Philippe Gillery ${ }^{1,3}$, Dr. Amar

Bennasroune ${ }^{1}$, Dr. Hervé Sartelet ${ }^{1}$, Dr. Muriel Lafargue ${ }^{2}$, Pr. Laurent Martiny ${ }^{1}$, Pr. Laurent

Duca $^{1}$, Dr. Pascal Maurice ${ }^{1}$

\section{(1)}

${ }^{1}$ UMR CNRS 7369 Matrice Extracellulaire et Dynamique Cellulaire (MEDyC), Université de Reims Champagne Ardenne (URCA), UFR Sciences Exactes et Naturelles, Reims, France.

${ }^{2}$ INSERM UMR1048 I2MC, Université Paul Sabatier, Toulouse, France.

${ }^{3}$ University Hospital of Reims, Department of Biochemistry-Pharmacology-Toxicology, Reims, France.

\section{*orresponding author:}

Dr Pascal Maurice, UMR CNRS/URCA 7369 MEDyC, Team 2 "Matrix aging and Vascular remodelling", UFR Sciences Exactes et Naturelles, Moulin de la Housse, BP1039, 51687 Reims cedex 2, France.

Phone: (+33) 326913275 / e-mail: pascal.maurice@univ-reims.fr

Short running title: Oseltamivir phosphate and vascular diseases

Sources of funding: This work was supported by funding from CNRS, URCA, and the

Fondation de France

Keywords: oseltamivir phosphate; sialidase; atherosclerosis; thrombosis; liver; inflammation 


\section{Abstract}

Desialylation, governed by sialidases or neuraminidases, is strongly implicated in a wide range of human disorders, and accumulative data show that inhibition of neuraminidases, such as NEU1 sialidase, may be useful for managing atherosclerosis. Several studies have reported promising effects of oseltamivir phosphate, a widely used anti-influenza sialidase inhibitor, on human cancer cells, inflammation and insulin resistance. In this study, we evaluated the effects of oseltamivir phosphate on atherosclerosis and thrombosis and potential liver toxicity in $\mathrm{LDLR}^{-/}$mice fed with high fat diet. Our results showed that oseltamivir phosphate significantly decreased plasma levels of LDL-cholesterol and elastin fragmentation in aorta. However, no effect was observed on both atherosclerotic plaque size in aortic roots and chemically-induced thrombosis in carotid arteries. Importantly, oseltamivir phosphate administration had adverse effects on the liver of mice and significantly increased mRNA expression levels of F4/80, IL-1 $\beta$, TGF- $\beta 1$, MMP-12 and collagen. Taken together, our findings suggest that oseltamivir phosphate has limited benefits on atherosclerosis and carotid thrombosis and may lead to side effects in the liver with increased inflammation and fibrosis.

(1)

(1)

45

\section{6}

\section{7}




\section{Introduction}

Human neuraminidases, or sialidases, are glycoside hydrolases which release terminal sialic acid residues from glycoproteins, glycolipids and oligosaccharides (1). Four types of mammalian neuraminidases (NEU), encoded by different genes, have been described with distinct substrate specificity and subcellular localization: NEU1 (2), NEU2 (3), NEU3 (4) and NEU4 (5). Sialidases have been implicated in a wide range of human disorders, including cancers, metabolic and cardiovascular diseases (6). Accumulative data highlighted sialidases as promising therapeutic targets, and modulating the activity of these enzymes may have beneficial effects in several pathologies.

Due to the lack of selective inhibitors, the vast majority of studies reported so far have used DANA (2,3- dehydro-2-deoxy-N-acetylneuraminic acid), a non-selective inhibitor of the four NEU isoenzymes, or oseltamivir phosphate (OP), an anti-influenza drug (7). OP is a prodrug which is converted by carboxyl esterase enzymes into the active oseltamivir carboxylate. OP is a sialic acid analogue which interacts with and blocks sialidase enzymes of the influenza virus, blocking their ability to release virions in the infected cells (8). Although OP demonstrates almost no appreciable inhibition of human sialidases $(9,10)$, contradictory results were obtained with this drug when tested on human cancer cells using both in vitro and in vivo models (11-15). It was also reported that OP reverses the epithelial to mesenchymal transition process and increases drug sensitivity of chemoresistant human cancer cells (16, 17). Moreover, in human monocytic cells, OP has been shown to decrease pro-inflammatory cytokines production in response to LPS $(18,19)$ or thymoquinone, derived from the nutraceutical black cumin oil (20). Both agonists activate toll-like receptors and trigger NFkB activation through NEU1 and NEU4, respectively. Finally, reported effects of OP on NEU1mediated regulation of insulin receptor (IR), phosphorylation of IR $\beta$ and insulin receptor substrate-1 (IRS-1) in response to insulin (21) and on IR transactivation by G-protein coupled 
receptors (22), suggest potential application of OP treatment in insulin resistance and type 2 diabetes.

Accumulative evidences also showed that neuraminidases inhibition, and more precisely inhibition of NEU1 sialidase, may be useful for managing atherosclerosis development (2325). Atherosclerosis induces the progressive luminal narrowing of arteries, which is the underlying cause of myocardial infarction and ischemic stroke. Formation of atherosclerotic plaques involve the accumulation of cholesterol and inflammatory cells in the sub-endothelial space of the vessel intima and is closely linked to extracellular matrix remodeling and elastic fibers degradation (26). However, evaluation of OP efficacy in animal models of atherosclerosis has never been reported so far. In addition, the use of OP in patients has been associated with adverse effects such as liver toxicity $(9,27-29)$, and the benefits versus harms of OP remains to be further evaluated before potential application in human for treatment of cancer, metabolic and cardiovascular diseases.

In the present paper, we evaluated the effects of OP administration on atherosclerosis and chemically-induced arterial thrombosis in $\mathrm{LDLR}^{-/-}$mice fed with a high fat diet (HFD). We assessed the effects of OP on plasma lipid parameters, elastin fragmentation in aorta, and liver inflammation and fibrosis. Whereas OP was shown to significantly reduce plasma LDLcholesterol levels and elastic fibers fragmentation in aorta, no benefit was observed on atherosclerosis development after 12 weeks of HFD, and on the time for complete carotid artery occlusion following $\mathrm{FeCl}_{3}$ injury. Importantly, adverse effects were observed in the liver of mice with increased inflammation and fibrosis. In conclusion, this study suggests that OP has limited effects on atherosclerosis and carotid thrombosis and that caution must be taken regarding its potential adverse effects on the liver. 


\section{Materials and methods}

102

103

104

105

106

107

108

109

110

111

112

113

114

115

116

117

118

119

120

121

122

123

124

125

\section{Animals}

All mouse experiments were approved by the Ethics Committee for Animal Welfare of the University of Reims Champagne-Ardenne (CEEA-RCA no 56) and the French Ministry of Research (APAFIS \# 5464-2016052416307263v4) being compliant with the European Directive 2010/63/UE. LDLR ${ }^{-/-}$(B6.129S7-Ldlr ${ }^{\mathrm{tm} 1 \mathrm{Her} / J}$ ) female mice were purchased from Charles River Laboratories (L'Arbresle, France). $\mathrm{LDLR}^{-/-}$mice (12 weeks-old) were fed for 12 weeks with HFD + $0.15 \%$ cholesterol or normal diet (ND) (Special Diet Services, DIETEX France Sarl, Argenteuil, France). The HFD was constituted by crude protein $22.6 \%$ (w/w), crude fat $23.0 \%(\mathrm{w} / \mathrm{w})$, crude fibers $4.6 \%(\mathrm{w} / \mathrm{w})$, Ash $4.2 \%(\mathrm{w} / \mathrm{w})$, carbohydrate $39.8 \%$ $(\mathrm{w} / \mathrm{w})$

\section{Oseltamivir phosphate}

OP capsules (75 mg, Roche) were reconstituted in sterile phosphate-buffered saline (PBS), and centrifuged at $1,000 \mathrm{rpm}$ for 10 minutes to obtain OP in the supernatant as previously reported (13). The stock-extracted OP solution had a concentration of $15 \mathrm{mg} / \mathrm{mL}$. OP was used at a concentration $(50 \mathrm{mg} / \mathrm{kg})$ that completely ablates tumor vascularization, tumor growth and spread to the lungs in a mouse model of heterotopic xenografts of MDA-MB-231 tumors (13) and twice lower than the one used by Gilmour et al in a mouse model of heterotopic xenografts of human pancreatic cancer (12). OP or vehicle were intraperitoneally injected each week, starting at the onset of HFD, and for 12 weeks.

\section{Intraperitoneal glucose tolerance test}

Glucose tolerance tests were performed according to Heikkinen et al (30). Briefly, 20\% aqueous glucose solution was administrated by intraperitoneal injection ( $2 \mathrm{mg}$ glucose/g body 
weight) in mice fasted for $4 \mathrm{~h}$. Glycemia was then measured from a blood drop taken from the tail of mice at baseline and 15, 30, 45, 60 and 90 min after glucose administration.

\section{Blood measurements}

At diet completion, mice were fasted for $6 \mathrm{~h}$ and blood was collected from the retro-orbital sinus into heparin tubes, centrifuged and plasma aliquots were kept at $-80^{\circ} \mathrm{C}$. Plasma glucose, insulin, total-, LDL-, and HDL-cholesterol, triglyceride and free fatty acids levels were measured by the Institut Clinique de la Souris (llkirch, France). Homeostatic model assessment (HOMA) for insulin resistance (HOMA-IR) was calculated according to (30): HOMA-IR = (FPI x FPG) / 22.5 where FPI was the fasting plasma insulin concentration $(\mu \mathrm{U} / \mathrm{mL})$ and FPG was the fasting plasma glucose concentration $(\mathrm{mmol} / \mathrm{L})$. Glycated haemoglobin $\left(\mathrm{HbA}_{1 \mathrm{c}}\right)$ was quantified by immunoassay using the DCA Vantage Analyzer (Siemens Healthcare, Erlangen, Germany).

\section{Quantification of elastin fragmentation in the aorta}

Mice were sacrificed by cervical dislocation, and aorta were collected rapidly and embedded within optimal cutting temperature (OCT) (Tissue-Tek) compound before placing them at $80^{\circ} \mathrm{C}$ until analysis. Cryosections $(10 \mu \mathrm{m})$ from aorta was visualized under an epifluorescence microscope (Olympus BX51WIF, Olympus France S.A.S., Rungis, France) connected to a digital camera (Olympus CAM-UC90). Elastin has autofluorescent properties and emits fluorescence at 500- $560 \mathrm{~nm}$ when excited at $488 \mathrm{~nm}(31)$.

\section{Aortic pulse wave velocity}

Doppler ultrasound (Indus Mouse Doppler System, Webster, TX) was performed under isoflurane anesthesia. Mice were placed supine on a heating board with legs secured to 
electrocardiographic electrodes. Doppler probes (10 and $20 \mathrm{MHz}$ ) were placed on the transverse aortic arch $(10 \mathrm{MHz})$ and abdominal aorta $(20 \mathrm{MHz})$ and the distance between the probes was determined with a precision caliper. Pre-ejection time, the time between the Rwave of the electrocardiogram and the foot of the Doppler signal, was determined for each site. Aortic PWV (aPWV) was calculated by dividing the distance $(\mathrm{cm})$ between the probes by the difference in pre-ejection times (milliseconds) of the thoracic and abdominal regions.

\section{Quantification of atherosclerotic lesions}

The hearts were prepared as described previously (32) and the atherosclerotic lesions were estimated according to Paigen et al. (33). Briefly, the hearts were washed in PBS and incubated in PBS at $4^{\circ} \mathrm{C}$ for $12 \mathrm{~h}$. Each heart was embedded in OCT compound and stored at $80^{\circ} \mathrm{C}$. One hundred sections of $10 \mu \mathrm{m}$ thickness were prepared from the top of the left ventricle, where the aortic valves were first visible, up to a position in the aorta where the valves were just disappearing from the field. After drying for $2 \mathrm{~h}$, the sections were stained with Oil Red O (ORO) and counterstained with Mayer's haematoxylin. After ORO staining, surface lesion areas were measured by computer assisted image quantification using the Leica QWin software (Leica Microsystems, Wetzlar, Germany). Images were captured with a Sony (Tokyo, Japan) 3CCD video camera.

\section{Mouse model of chemically-induced arterial thrombosis}

The carotid artery of anesthetized mice was isolated from the surrounding tissue, and placed forward of a small piece of non-transparent black plastic film to prevent fluorescence interferences by underlying structures during the visualization of blood flow. Rhodamine $6 \mathrm{G}$ $(1 \mathrm{mg} / \mathrm{mL})$ was then retro-orbitally injected. Redox-induced vascular endothelial injury was achieved by applying a piece of filter paper $(2 \times 1 \mathrm{~mm})$ saturated with a $7.5 \%$ ferric chloride 
$\left(\mathrm{FeCl}_{3}\right)$ solution directly on the carotid artery for 1 minute. The filter paper was then removed, and thrombus formation was observed in real time using an epifluorescence microscope (Olympus BX51WIF, Olympus France S.A.S., Rungis, France) connected to a digital camera (Olympus CAM-UC90). Time for complete thrombotic occlusion was defined as blood flow being stopped for at least 30 seconds.

\section{RNA Extraction and quantitative RT-PCR}

For each sample, mouse livers were recovered, flash frozen, and a fraction was grinded in 1 $\mathrm{mL}$ of Qiazol (Qiagen, France) with an Ultra Thurax (Imlab, France). Total RNA was extracted using RNeasy Lipid Tissue Mini Kit (Qiagen, France), and 250 ng were reverse transcripted using the Verso cDNA Synthesis Kit (ThermoFisher Scientific, France) according to the manufacturer's instructions. The transcript levels were determined by realtime quantitative PCR using the CFX $96{ }^{\mathrm{TM}}$ Real-Time System (Biorad, Hercules, CA, USA) and the SYBR Green Master Mix PCR kit as recommended by the manufacturer (ThermoFisher Scientific, France). PCR reactions were carried out in duplicates in 96-well plates $(15 \mu \mathrm{L}$ per well) in a buffer containing $1 x$ SYBR Green mix (including Taq polymerase, dNTPs, SYBR Green dye), $280 \mathrm{nM}$ forward and reverse primers and 1:10 dilution of reverse transcript RNA. After denaturation at $95^{\circ} \mathrm{C}$ for $15 \mathrm{~min}$, amplification occurred in a two-step procedure: $15 \mathrm{~s}$ of denaturation at $95^{\circ} \mathrm{C}$ and $1 \mathrm{~min}$ of annealing/extension at $60^{\circ} \mathrm{C}$, with a total of 30 cycles. Identical thermal cycling conditions were used for all targets. Specific primers were designed using the NCBI Primer Blast interface and are presented in Table 1. PCR efficiency of the primer sets was calculated by performing real-time PCR on serial dilutions. For each experiment, PCR reactions were performed in duplicate and 3 independent experiments were analyzed. Relative gene expression was determined with the formula fold induction: $2^{-\Delta \Delta \mathrm{Ct}}$, where $\Delta \Delta C \mathrm{t}=(C \mathrm{t}$ GI 
201 [unknown sample]-Ct GI [reference sample])-( $C \mathrm{t}$ reference genes [unknown sample]- $C \mathrm{t}$

202 reference genes [reference sample]). GI is the gene of interest. RPS26 and 36B4 are used as 203 reference genes. The reference samples are ND mice and the unknown samples are HFD 204 mice.

\section{Statistical analysis}

207 Data were expressed as mean \pm standard error of the mean (SEM). In vitro and ex vivo parametric data were compared with two-tailed Student $t$-test. In vivo non-parametric data were compared with two-tailed Mann-Whitney $U$ test. Multiple comparisons were done by one-way ANOVA followed by Dunnett's test. P-value $\leq 0.05$ was considered statistically significant. Statistical analysis was performed using GraphPad Prism (GraphPad Software, La Jolla, USA). 


\section{Results}

226

227

\section{LDLR $^{-/-}$mice fed with a HFD for 12 weeks display type 2 diabetes and dyslipidemia}

$\mathrm{LDLR}^{-/-}$mice were fed with HFD (HFD mice) and compared to ND-fed mice (ND mice). After 12 weeks of diet, HFD mice showed a significant higher body weight that ND mice (31.9 \pm 1.1 vs 23.3 \pm 0.9$)$ (Fig 1A) and were intolerant to glucose (Fig 1B). In addition, HFD mice showed significant increased blood glucose $(8.40 \pm 0.78$ vs $4.33 \pm 0.18 \mathrm{mmol} / \mathrm{L}), \mathrm{HbA}_{1 \mathrm{c}}$ $(25.0 \pm 0.4$ vs $20.4 \pm 1.4 \mathrm{mmol} / \mathrm{mol})$, and insulin $(0.128 \pm 0.024$ vs $0.057 \pm 0.015 \mu \mathrm{g} / \mathrm{L})$ concentrations (Fig 1C, D, E). The HOMA-IR index was also significantly increased in HFD mice $(1.02 \pm 0.12$ vs $0.34 \pm 0.09)$ (Fig $1 \mathrm{~F})$. Total cholesterol $(24.57 \pm 1.50$ vs $15.88 \pm 0.79$ $\mathrm{mmol} / \mathrm{L}), \mathrm{HDL}-(5.07 \pm 0.16$ vs $3.22 \pm 0.12 \mathrm{mmol} / \mathrm{L})$ and LDL-cholesterol $(15.65 \pm 0.87 v s$ $10.15 \pm 0.53 \mathrm{mmol} / \mathrm{L})$ concentrations were also significantly increased compared to ND mice (Fig 1G, H, I). Unexpectedly, no significant difference was found in free fatty acids and triglycerides plasma levels between HFD and ND mice (Supplemental Digital Content 1). Taken together, these results demonstrated that HFD mice are dyslipidemic and present all the characteristics of type 2 diabetes (hyperglycemia, glucose intolerance, insulin resistance).

\section{HFD mice display elastic fibers fragmentation and increased aortic pulse wave velocity}

We next evaluated the impact of HFD on extracellular matrix remodeling in the aorta of mice by measuring elastic fibers fragmentation. Elastin has autofluorescent properties and emits fluorescence at 500- $560 \mathrm{~nm}$ when excited at $488 \mathrm{~nm}$ (31) (Fig 2A). In HFD mice, the main number of breaks per elastic lamella was significantly increased compared to ND mice (1.4 \pm 0.1 vs $0.3 \pm 0.1$ breaks, Fig 2B). Moreover, the repartition of the number of breaks per elastic lamella was also strongly different. In ND mice, up to 2 breaks per elastic lamella was observed with the following repartition: no break (72\%), 1 break (23\%) and 2 breaks (5\%) 
(Fig 2C). In contrast, up to 6 breaks per elastic lamella was observed in HFD mice with the following repartition: no break (31\%), 1 (30\%), $2(18 \%), 3(12 \%), 4(6 \%), 5(2 \%)$ and 6 breaks $(<1 \%)$ (Fig 2C). Taken together, these results demonstrated that HFD induced vascular extracellular matrix remodeling and fragmentation of elastic fibers in the aorta of mice.

Finally, we monitored the evolution of aPWV in HFD mice (Fig 2D). At baseline, aPWV was calculated at $0.365 \pm 0.024 \mathrm{~cm} / \mathrm{ms}$. No major modification was observed between baseline and 9 weeks of HFD $(0.377 \pm 0.024 \mathrm{~cm} / \mathrm{ms})$. After 12 weeks, aPWV was significantly increased to $0.449 \pm 0.021 \mathrm{~cm} / \mathrm{ms}$ in HFD mice.

\section{Administration of oseltamivir phosphate in HFD mice has limited effects on type 2}

\section{diabetes and dyslipidemia}

The impact of OP administration was next evaluated in $\mathrm{LDLR}^{-/-}$mice fed with the same HFD during 12 weeks. OP (50 mg/kg, + OP) or vehicle (- OP) were administrated weekly by intraperitoneal injections, starting at the onset of HFD. At this concentration $(50 \mathrm{mg} / \mathrm{kg})$, OP was previously shown to completely ablate tumor vascularization, tumor growth and spread to the lungs in a mouse model of heterotopic xenografts of MDA-MB-231 tumors (13). As shown in Fig 3A, B, C, no significant difference was observed on glycemia, insulinemia, and HOMA-IR index between OP- and vehicle-treated groups, although a trend for increased insulinemia and HOMA-IR index was noted. Body weights (Fig $3 \mathrm{G}$ ), $\mathrm{HbA}_{1 \mathrm{c}}$, free fatty acids and triglycerides concentrations (Supplemental Digital Content 2) were also comparable between OP- and vehicle-treated mice. When considering plasma cholesterol (Fig 3D, E, F), a significant decrease of total-cholesterol concentrations was observed in OP-treated group when compared to vehicle-treated group $(19.95 \pm 1.29$ vs $24.57 \pm 1.50 \mathrm{mmol} / \mathrm{L})$. This was associated with a significant reduction of LDL-cholesterol concentrations in OP-treated mice (12.98 \pm 0.78 vs $15.65 \pm 0.67 \mathrm{mmol} / \mathrm{L})$. 
Administration of oseltamivir phosphate in HFD mice decreases elastic fibers

fragmentation without modification of pulse wave velocity

277

We next evaluated the effects of OP treatment on elastic fibers fragmentation and aPWV. As shown in Fig 2B, HFD induces a significant increase in elastic fibers breaks compared to ND. In HFD mice, OP administration was able to significantly decrease the main number of breaks per elastic lamella compared to vehicle-treated mice $(0.9 \pm 0.1$ vs $1.7 \pm 0.3$, Fig $4 \mathrm{~A})$. This was associated with major differences in the repartition of the number of breaks per elastic lamella (Fig 4B). In vehicle-treated mice, up to 7 breaks per elastic lamella was observed. In OPtreated mice, no more than 4 breaks were observed. The repartition of elastin breaks was as follows for the vehicle-treated group: no break (18\%), 1 (39\%), $2(21 \%), 3(14 \%), 6(4 \%)$ and 7 breaks (4\%). For the OP-treated group, the repartition was no break (40\%), 1 (35\%), 2 (17\%), $3(6 \%)$ and 4 breaks (2\%). However, no major impact on the aPWV parameter was observed between the 2 groups (Fig 4C).

\section{Administration of oseltamivir phosphate in HFD mice has no effect on atherosclerosis} and carotid thrombosis

After 12 weeks of HFD, $\mathrm{LDLR}^{-/-}$mice developed large atherosclerotic lesions in aortic roots (Fig 5A, left panels). After ORO staining and quantification, the mean lesion size was estimated at $0.36 \pm 0.03 \mathrm{~mm}^{2}$ for vehicle-treated mice and $0.41 \pm 0.02 \mathrm{~mm}^{2}$ for OP-treated mice (Fig 5A, right panels). Together, these data demonstrated that, in our experimental conditions, OP treatment had no effect on atherosclerosis development. We also evaluated the effects of OP treatment on arterial thrombosis by using the $\mathrm{FeCl}_{3}$-induced carotid artery thrombosis model (34). Thrombosis was triggered by $7.5 \% \mathrm{FeCl}_{3}$ in both vehicle- and OP-treated HFD mice and time to complete thrombotic occlusion was monitored using real-time 
videomicroscopy and defined as an observed arrest in blood flow during at least 30 seconds.

No difference in the mean occlusion time was observed between vehicle- and OP-treated mice $(8.5 \pm 0.4$ vs $8.9 \pm 0.5 \mathrm{~min})$ (Fig 5B). Although not significant, a trend for a decrease of the number of embols released during thrombus formation was observed in the OP-treated group (11.5 \pm 3.5 vs $5.9 \pm 1.4$ embols) (Fig 5C). Taken together, these data demonstrated that, in our experimental conditions, OP treatment had no effect on carotid thrombosis but may lead to reduced embolization.

\section{Administration of oseltamivir phosphate in HFD mice induces liver inflammation and}

\section{fibrosis}

After 12 weeks of HFD, the liver weight of $\mathrm{LDLR}^{-/-}$mice had more than doubled compared to mice fed with ND diet $(1.34 \pm 0.07$ vs $0.61 \pm 0.19 \mathrm{~g})$. No difference was observed between vehicle- and OP-treated HFD mice (Fig 6A). To evaluate potential toxic effects of OP on the liver, expression of different inflammation (F4/80, IL-1 $\beta$, TGF- $\beta 1)$ and extracellular matrix remodeling (MMP-2, -9, -12, collagen) markers were assessed by quantitative RT-PCR. As shown in Fig 6B, $\mathrm{LDLR}^{-/-}$mice fed with HFD showed a moderate but significant increase by 1.6- and 1.3-fold of IL-1 $\beta$ and TGF- $\beta 1$ mRNA expression levels, respectively, compared to ND mice. No significant difference was observed for the macrophage F4/80 marker. In OPtreated HFD mice, F4/80, IL-1 $\beta$, TGF- $\beta 1$ mRNA expression levels were further significantly increased by 1.7-, 1.7- and 1.8-fold, respectively, compared to vehicle-treated HFD mice (Fig 6B). When considering markers of extracellular matrix remodeling, HFD mice induced a significant increase by 30-fold of MMP-12 mRNA expression levels compared to ND mice, and no significant difference was observed for MMP-2, -9 and type I collagen (alpha 1 chain) (Fig 6C). However, OP treatment further significantly increased by 1.7- and 2.5-fold MMP-12 and collagen mRNA expression levels, respectively, compared to vehicle-treated HFD mice 
324 (Fig 6C). Together, these results showed that OP administration to HFD-fed LDLR ${ }^{-/}$mice

325 could trigger liver inflammation, extracellular matrix remodeling and fibrosis.

326

327

328

329

330

331

332

333

334

335

336

337

338

339

340

341

342

343

344

345

346

347

348 


\section{Discussion}

Together with zanimivir, OP form the basis of clinically available flu antiviral therapeutics (35). Whereas viral sialidase inhibitors have also broad specificity for bacterial NEUs, studies that have assessed the activity of zanamivir and OP against human NEUs (hNEUs) have reported weaker efficacy $(9,10)$. In a recent study, $\mathrm{IC}_{50}$ values for $\mathrm{OP}$ were estimated at more than $500 \mu \mathrm{M}$ against the four hNEUs, and the only viral NEU inhibitor tested with notable activity against hNEU2, hNEU3 and hNEU4 was zanamivir (10). However, despite this low inhibitory potency of OP for hNEUs, several reports have evaluated its use as potential inhibitor of hNEU1 sialidase activity and related signaling pathways in in vitro and in vivo studies. Among the four hNEUs described so far, NEU1 is indeed the most studied sialidase. Firstly identified in lysosomes (2), NEU1 is also found at the plasma membrane of cells and regulates a myriad of membrane receptors by desialylation resulting in either activation or inhibition of the receptors (36-44). At the plasma membrane, NEU1 also associates with the elastin-binding protein (EBP) and the carboxypeptidase protective protein/cathepsin A (PPCA) forming the elastin receptor complex (ERC) (45). NEU1 is known to be required for elastogenesis and signal transduction through this receptor (45-47), and for the biological effects mediated by the elastin-derived peptides $(48,49)$.

Implication of NEU1 in atherosclerosis is supported by different studies. High expression of NEU1 has been reported in peripheral blood mononuclear cells of patients with acute myocardial infarction and in macrophages present in the intima layer, in calcified regions and within the adventitia of the plaque region in human carotid arteries (24). Enhanced NEU1 expression induces a pro-inflammatory phenotype, triggers production and release of cytokines and chemokines in monocytes, and promotes phagocytosis and cytokine expression in macrophages (24). In human macrophages, NEU1 also modulates the sialylation level of CD36 receptors and enhances oxidized LDL uptake in response to elastin-derived peptides 
(41). Direct evidence for NEU1 involvement in atherosclerosis came from the works of Gayral et al (23). Using chimeric LDLR ${ }^{-/-}$mice devoid of NEU1 activity in the haematopoietic lineage, Gayral et al showed decreased atheroma plaque size progression in aortic sinus and leucocytes infiltration. Moreover, in $\mathrm{ApoE}^{-/-}$mice expressing hypomorphic levels of NEU1 (Neul ${ }^{\text {hypo }} \mathrm{Apoe}^{-/-}$), White et al have reported reduced serum levels of verylow-density lipoprotein (VLDL) and LDL-cholesterol, decreased infiltration of inflammatory cells into lesions, and significant reduction of atherosclerosis (25). Consistent with these findings, administration of the broad spectrum sialidase inhibitor DANA had anti-atherogenic effect in the $\mathrm{ApoE}^{-/-}$mice (25). Taken together, all these studies suggest that targeting NEU1 expression or catalytic activity may represent a promising target for managing atherosclerosis. In light of these promising data, we evaluated in this study the effects of OP administration on atherosclerosis development in HFD-fed LDLR ${ }^{-/-}$mice, at a concentration $(50 \mathrm{mg} / \mathrm{kg})$ that completely ablates tumor vascularization, tumor growth and spread to the lungs in a mouse model of heterotopic xenografts of MDA-MB-231 tumors (13) and twice lower than the one used by Gilmour et al in a mouse model of heterotopic xenografts of human pancreatic cancer (12). From the results presented here, it is suggested that OP treatment has no protective effect on atherosclerosis development since comparable atheroma plaque size was observed in aortic sinus of vehicle- and OP-treated LDLR ${ }^{-/-}$mice. If these contradictory results rely on differences between diet formulation, diet duration and/or mice genotype remain to be further evaluated. In the present study, $\mathrm{LDLR}^{-/-}$mice were fed for 12 weeks with high-fat, highcarbohydrate diet, and one may consider that the protocol used here was too drastic to see any potential benefit of OP treatment. Moreover, OP was administrated weekly and not daily as described previously (13), and one may speculate that the time between two injections has to be shorten. However, using this protocol, OP was shown to be effective in reducing plasma LDL-cholesterol levels, in agreement with the works of White et al showing that $\mathrm{ApoE}^{-/-}$mice 
expressing hypomorphic levels of NEU1 had reduced serum levels of LDL-cholesterol (25). Taken together, these results show that inhibition of NEUs by OP modulates lipoprotein metabolism and reduces LDL-cholesterol levels. Modification of sialic acids on apolipoproteins is known to affect lipoprotein metabolism (50-52), and advanced desialylation of LDL by sialidases is considered as a primary step of atherogenic modification of lipids (53).

Here, we also observed that OP-treated HFD mice are prone to develop insulin resistance. Even if the difference failed to be significant between vehicle- and OP-treated groups, a near 2-fold increase of the HOMA-IR index was observed for OP-treated mice. Importantly, insulin receptor activation has been shown to be under the regulation of NEU1 $(37,38)$ and mice deficient in NEU1 rapidly develop glucose intolerance and insulin resistance after being fed with HFD (38). Further works have uncovered a crosstalk between NEU1 and MMP-9 in alliance with G-protein coupled receptor (GPCR), which was essential for insulin-induced IR activation and cellular signaling $(21,22)$.

Another interesting finding from this study comes from the protective effect of OP on elastic fibers integrity in aorta. Elastic fibers are fundamental for mechanical properties of the arterial wall, and elastic fibers fragmentation has major consequences on atherosclerosis progression (26). Degradation of elastin involves elastases such as MMPs (MMP-2, -7, -9, -12), cysteine cathepsins (L, S, K, V), and/or the neutrophil elastase, (54). Whether OP reduces expression or activity of elastases in aorta remains to be further evaluated. Damaged or degraded elastic fibers are generally not repaired and replaced by collagens and proteoglycans that stiffen the arterial wall. Even if no effect was observed on aPWV, OP treatment may help in limiting arterial stiffness occurring with atherosclerosis. Decreasing elastin fragmentation may also limit the generation of elastin-degradation products, which are critical players of atherosclerosis development (26). 
One major issue of atherosclerosis is plaque rupture and thrombosis. Since desialylation regulates platelet activation and aggregation $(55,56)$, we hypothesized that OP treatment may decrease atherothrombosis. However, we did not observe any difference in our chemicallyinduced carotid thrombosis model between vehicle- and OP-treated groups. In contrast, and even if the difference failed to be significant, a near 2-fold decrease of the number of embols released during thrombus formation was observed in OP-treated HFD mice. These results, that remain to be further confirmed, may suggest that OP could reduce embolization and hence increase thrombus stability.

The use of OP in patients has been associated with adverse effects such as nausea, vomiting, psychiatric effects, renal events (57), and liver toxicity (9, 27-29). Here, we report that OP treatment has adverse effects on the liver and significantly increased mRNA expression levels of typical inflammation markers such as the pan-marker of murine macrophages F4/80, and the pro-inflammatory cytokines IL- $1 \beta$ and TGF- $\beta 1$. Together with increase in mRNA expression levels of MMP-12 and type I collagen, our results suggest that OP treatment also triggers ECM remodeling in the liver and fibrogenic responses. Long-term consequences on potential steatohepatitis development associated with OP treatment remain to be evaluated.

In conclusion, the present study shows that, in these experimental settings, OP treatment has limited effects on atherosclerosis and related thrombotic events in $\mathrm{LDLR}^{-/-}$mice. Although higher concentrations and daily injections have to be considered before stating that OP has no protective effect on atherosclerosis and thrombosis, these data suggest that caution must be taken concerning its side effects on liver. Even if promising effects of OP have been reported in different animal models of human diseases, benefits versus harms of OP remains to be further evaluated before potential application in human. 


\section{Acknowledgements}

450 This work was supported by funding from CNRS, URCA, and the Fondation de France. We

451 thank Jean-Luc Breda for animal handling and Caroline Fichel for aorta cryosections.

452

453 Conflict of interest

454 The authors declare that they have no conflict interest.

455

456

\section{Author contributions}

457

458

459

460

461

462

463

464

465

466

467

468

469

470

471

472

PM conceived, designed, and supervised the study. LD, SB and ML helped in designing the study. OB, AW, TS, EV, CS, AF, SG, AH, SB, BRC and PM performed the experiments. AB, HS, SJ, PG and LM contributed to discussion. PM and LD were involved in writing the manuscript and PM and CS prepared the figures.

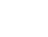




\section{References}

474 1. Miyagi T, Yamaguchi K. Mammalian sialidases: physiological and pathological roles 475 in cellular functions. Glycobiology. 2012;22(7):880-96.

476 2. Bonten E, van der Spoel A, Fornerod M, Grosveld G, d'Azzo A. Characterization of 477 human lysosomal neuraminidase defines the molecular basis of the metabolic storage disorder 478 sialidosis. Genes Dev. 1996;10(24):3156-69.

479 3. Monti E, Preti A, Rossi E, Ballabio A, Borsani G. Cloning and characterization of 480 NEU2, a human gene homologous to rodent soluble sialidases. Genomics. 1999;57(1):137-43.

481 4. Wada T, Yoshikawa Y, Tokuyama S, Kuwabara M, Akita H, Miyagi T. Cloning, 482 expression, and chromosomal mapping of a human ganglioside sialidase. Biochem Biophys 483 Res Commun. 1999;261(1):21-7.

484 5. Monti E, Bassi MT, Bresciani R, Civini S, Croci GL, Papini N, et al. Molecular 485 cloning and characterization of NEU4, the fourth member of the human sialidase gene family. 486 Genomics. 2004;83(3):445-53.

487 6. Glanz VY, Myasoedova VA, Grechko AV, Orekhov AN. Sialidase activity in human 488 pathologies. Eur J Pharmacol. 2019;842:345-50.

489 7. Bassetti M, Castaldo N, Carnelutti A. Neuraminidase inhibitors as a strategy for 490 influenza treatment: pros, cons and future perspectives. Expert Opin Pharmacother. $491 \quad 2019 ; 20(14): 1711-8$.

492 8. Wilson JC, von Itzstein M. Recent strategies in the search for new anti-influenza 493 therapies. Curr Drug Targets. 2003;4(5):389-408.

494 9. Hata K, Koseki K, Yamaguchi K, Moriya S, Suzuki Y, Yingsakmongkon S, et al. 495 Limited inhibitory effects of oseltamivir and zanamivir on human sialidases. Antimicrob 496 Agents Chemother. 2008;52(10):3484-91. 
10. Richards MR, Guo T, Hunter CD, Cairo CW. Molecular dynamics simulations of viral neuraminidase inhibitors with the human neuraminidase enzymes: Insights into isoenzyme selectivity. Bioorg Med Chem. 2018;26(19):5349-58.

11. Abdulkhalek S, Geen OD, Brodhagen L, Haxho F, Alghamdi F, Allison S, et al. Transcriptional factor snail controls tumor neovascularization, growth and metastasis in mouse model of human ovarian carcinoma. Clin Transl Med. 2014;3(1):28.

12. Gilmour AM, Abdulkhalek S, Cheng TS, Alghamdi F, Jayanth P, O'Shea LK, et al. A novel epidermal growth factor receptor-signaling platform and its targeted translation in pancreatic cancer. Cell Signal. 2013;25(12):2587-603.

13. Haxho F, Allison S, Alghamdi F, Brodhagen L, Kuta VE, Abdulkhalek S, et al.

Oseltamivir phosphate monotherapy ablates tumor neovascularization, growth, and metastasis in mouse model of human triple-negative breast adenocarcinoma. Breast Cancer (Dove Med Press). 2014;6:191-203.

14. Haxho F, Neufeld RJ, Szewczuk MR. Neuraminidase-1: a novel therapeutic target in multistage tumorigenesis. Oncotarget. 2016;7(26):40860-81.

15. Wood K, Szewczuk MR, Rousseau D, Neufeld RJ. Oseltamivir phosphate released from injectable Pickering emulsions over an extended term disables human pancreatic cancer cell survival. Oncotarget. 2018;9(16):12754-68.

16. O'Shea LK, Abdulkhalek S, Allison S, Neufeld RJ, Szewczuk MR. Therapeutic targeting of Neu1 sialidase with oseltamivir phosphate (Tamiflu(R)) disables cancer cell survival in human pancreatic cancer with acquired chemoresistance. Onco Targets Ther. 2014;7:117-34.

17. Thulasiraman P, Kerr K, McAlister K, Hardisty S, Wistner A, McCullough I. Neuraminidase 1 regulates proliferation, apoptosis and the expression of Cadherins in mammary carcinoma cells. Mol Cell Biochem. 2019;462(1-2):207-15. 
18. Abdulkhalek S, Szewczuk MR. Neu1 sialidase and matrix metalloproteinase-9 crosstalk regulates nucleic acid-induced endosomal TOLL-like receptor-7 and -9 activation, cellular signaling and pro-inflammatory responses. Cell Signal. 2013;25(11):2093-105. 19. Amith SR, Jayanth P, Franchuk S, Siddiqui S, Seyrantepe V, Gee K, et al. Dependence of pathogen molecule-induced toll-like receptor activation and cell function on Neu1 sialidase. Glycoconj J. 2009;26(9):1197-212.

20. Finlay TM, Abdulkhalek S, Gilmour A, Guzzo C, Jayanth P, Amith SR, et al. Thymoquinone-induced Neu4 sialidase activates NFkappaB in macrophage cells and proinflammatory cytokines in vivo. Glycoconj J. 2010;27(6):583-600.

21. Alghamdi F, Guo M, Abdulkhalek S, Crawford N, Amith SR, Szewczuk MR. A novel insulin receptor-signaling platform and its link to insulin resistance and type 2 diabetes. Cell Signal. 2014;26(6):1355-68.

22. Haxho F, Haq S, Szewczuk MR. Biased G protein-coupled receptor agonism mediates Neu1 sialidase and matrix metalloproteinase- 9 crosstalk to induce transactivation of insulin receptor signaling. Cell Signal. 2018;43:71-84.

23. Gayral S, Garnotel R, Castaing-Berthou A, Blaise S, Fougerat A, Berge E, et al. Elastin-derived peptides potentiate atherosclerosis through the immune Neu1-PI3Kgamma pathway. Cardiovasc Res. 2014;102(1):118-27.

24. Sieve I, Ricke-Hoch M, Kasten M, Battmer K, Stapel B, Falk CS, et al. A positive feedback loop between IL-1beta, LPS and NEU1 may promote atherosclerosis by enhancing a pro-inflammatory state in monocytes and macrophages. Vascul Pharmacol. 2018;103-105:1628.

25. White EJ, Gyulay G, Lhotak S, Szewczyk MM, Chong T, Fuller MT, et al. Sialidase down-regulation reduces non-HDL cholesterol, inhibits leukocyte transmigration, and attenuates atherosclerosis in ApoE knockout mice. J Biol Chem. 2018;293(38):14689-706. 
26. Duca L, Blaise S, Romier B, Laffargue M, Gayral S, El Btaouri H, et al. Matrix ageing and vascular impacts: focus on elastin fragmentation. Cardiovasc Res. 2016;110(3):298-308. 27. El-Sayed WM, Al-Kahtani MA. Potential adverse effects of oseltamivir in rats: males are more vulnerable than females. Can J Physiol Pharmacol. 2011;89(9):623-30.

28. Fang S, Qi L, Zhou N, Li C. Case report on alimentary tract hemorrhage and liver injury after therapy with oseltamivir: A case report. Medicine (Baltimore). 2018;97(38):e12497.

29. Hama R. The mechanisms of delayed onset type adverse reactions to oseltamivir. Infect Dis (Lond). 2016;48(9):651-60.

30. Heikkinen S, Argmann CA, Champy MF, Auwerx J. Evaluation of glucose homeostasis. Curr Protoc Mol Biol. 2007; Chapter 29:Unit 29B 3.

31. Monici M. Cell and tissue autofluorescence research and diagnostic applications. Biotechnol Annu Rev. 2005;11:227-56.

32. Fougerat A, Pan X, Smutova V, Heveker N, Cairo CW, Issad T, et al. Neuraminidase 1 activates insulin receptor and reverses insulin resistance in obese mice. Mol Metab. 2018;12:76-88.

33. Paigen B, Morrow A, Holmes PA, Mitchell D, Williams RA. Quantitative assessment of atherosclerotic lesions in mice. Atherosclerosis. 1987;68(3):231-40.

34. Li W, McIntyre TM, Silverstein RL. Ferric chloride-induced murine carotid arterial injury: A model of redox pathology. Redox Biol. 2013;1:50-5.

35. von Itzstein M. The war against influenza: discovery and development of sialidase inhibitors. Nat Rev Drug Discov. 2007;6(12):967-74.

36. Amith SR, Jayanth P, Franchuk S, Finlay T, Seyrantepe V, Beyaert R, et al. Neu1 desialylation of sialyl alpha-2,3-linked beta-galactosyl residues of TOLL-like receptor 4 is essential for receptor activation and cellular signaling. Cell Signal. 2010;22(2):314-24. 
37. Blaise S, Romier B, Kawecki C, Ghirardi M, Rabenoelina F, Baud S, et al. Elastinderived peptides are new regulators of insulin resistance development in mice. Diabetes. 2013;62(11):3807-16.

38. Dridi L, Seyrantepe V, Fougerat A, Pan X, Bonneil E, Thibault P, et al. Positive regulation of insulin signaling by neuraminidase 1. Diabetes. 2013;62(7):2338-46.

39. Hinek A, Bodnaruk TD, Bunda S, Wang Y, Liu K. Neuraminidase-1, a subunit of the cell surface elastin receptor, desialylates and functionally inactivates adjacent receptors interacting with the mitogenic growth factors PDGF-BB and IGF-2. Am J Pathol. 2008;173(4):1042-56.

40. Jayanth P, Amith SR, Gee K, Szewczuk MR. Neu1 sialidase and matrix metalloproteinase-9 cross-talk is essential for neurotrophin activation of Trk receptors and cellular signaling. Cell Signal. 2010;22(8):1193-205.

41. Kawecki C, Bocquet O, Schmelzer CEH, Heinz A, Ihling C, Wahart A, et al. Identification of CD36 as a new interaction partner of membrane NEU1: potential implication in the pro-atherogenic effects of the elastin receptor complex. Cell Mol Life Sci. 2019;76(4):791-807.

42. Lee C, Liu A, Miranda-Ribera A, Hyun SW, Lillehoj EP, Cross AS, et al. NEU1 sialidase regulates the sialylation state of CD31 and disrupts CD31-driven capillary-like tube formation in human lung microvascular endothelia. J Biol Chem. 2014;289(13):9121-35.

43. Lillehoj EP, Hyun SW, Feng C, Zhang L, Liu A, Guang W, et al. NEU1 sialidase expressed in human airway epithelia regulates epidermal growth factor receptor (EGFR) and MUC1 protein signaling. J Biol Chem. 2012;287(11):8214-31.

44. Uemura T, Shiozaki K, Yamaguchi K, Miyazaki S, Satomi S, Kato K, et al. Contribution of sialidase NEU1 to suppression of metastasis of human colon cancer cells through desialylation of integrin beta4. Oncogene. 2009;28(9):1218-29. 
45. Hinek A, Pshezhetsky AV, von Itzstein M, Starcher B. Lysosomal sialidase (neuraminidase-1) is targeted to the cell surface in a multiprotein complex that facilitates elastic fiber assembly. J Biol Chem. 2006;281(6):3698-710.

46. Duca L, Blanchevoye C, Cantarelli B, Ghoneim C, Dedieu S, Delacoux F, et al. The elastin receptor complex transduces signals through the catalytic activity of its Neu-1 subunit. J Biol Chem. 2007;282(17):12484-91.

47. Rusciani A, Duca L, Sartelet H, Chatron-Colliet A, Bobichon H, Ploton D, et al. Elastin peptides signaling relies on neuraminidase-1-dependent lactosylceramide generation. PLoS One. 2010;5(11):e14010.

48. Bennasroune A, Romier-Crouzet B, Blaise S, Laffargue M, Efremov RG, Martiny L, et al. Elastic fibers and elastin receptor complex: Neuraminidase-1 takes the center stage. Matrix Biol. 2019;84:57-67.

49. Wahart A, Hocine T, Albrecht C, Henry A, Sarazin T, Martiny L, et al. Role of elastin peptides and elastin receptor complex in metabolic and cardiovascular diseases. FEBS J. 2019;286(15):2980-93.

50. Filipovic I, Schwarzmann G, Mraz W, Wiegandt H, Buddecke E. Sialic-acid content of low-density lipoproteins controls their binding and uptake by cultured cells. Eur J Biochem. 1979;93(1):51-5.

51. Millar JS. The sialylation of plasma lipoproteins. Atherosclerosis. 2001;154(1):1-13.

52. Orekhov AN, Tertov VV, Sobenin IA, Smirnov VN, Via DP, Guevara J, Jr., et al. Sialic acid content of human low density lipoproteins affects their interaction with cell receptors and intracellular lipid accumulation. J Lipid Res. 1992;33(6):805-17.

53. Zhang C, Chen J, Liu Y, Xu D. Sialic acid metabolism as a potential therapeutic target of atherosclerosis. Lipids Health Dis. 2019;18(1):173. 
621 54. Maurice P, Blaise S, Gayral S, Debelle L, Laffargue M, Hornebeck W, et al. Elastin

622 fragmentation and atherosclerosis progression: the elastokine concept. Trends Cardiovasc

623 Med. 2013;23(6):211-21.

624 55. Kullaya V, de Jonge MI, Langereis JD, van der Gaast-de Jongh CE, Bull C, Adema

625 GJ, et al. Desialylation of Platelets by Pneumococcal Neuraminidase A Induces ADP-

626 Dependent Platelet Hyperreactivity. Infect Immun. 2018;86(10).

627 56. van der Wal DE, Davis AM, Mach M, Marks DC. The role of neuraminidase 1 and 2

628 in glycoprotein Ibalpha-mediated integrin alphaIlbbeta3 activation. Haematologica.

$629 \quad 2020 ; 105(4): 1081-94$.

630 57. Jefferson T, Jones MA, Doshi P, Del Mar CB, Hama R, Thompson MJ, et al.

631 Neuraminidase inhibitors for preventing and treating influenza in healthy adults and children.

632 Cochrane Database Syst Rev. 2014(4):CD008965.

633

634

635

636

637

638

639

640

641

642

643

644

645 


\section{Figure legends}

647

648

649

650

651

652

653

654

655

656

657

658

659

660

661

662

663

664

665

666

667

668

669

670

Figure 1. Impact of HFD on body weight gain and metabolic parameters in LDLR ${ }^{-/-}$ mice.

Body weight (A), glucose tolerance test (B), plasma glucose (C), $\operatorname{HbA}_{1 \mathrm{C}}(\mathrm{D})$ plasma insulin (E), HOMA-IR index (F), and plasma total- (G), HDL- (H), and LDL- (I) cholesterol concentrations were measured from fasted $\mathrm{LDLR}^{-/-}$mice. White bars (A, C-I) and white circles (B) correspond to mice fed with normal diet (ND), and black bars (A, C-I) and black squares (B) correspond to mice fed with HFD. Data represent the mean \pm SEM of 4 to 5 mice. $(*, \mathrm{p}<0.05 ; * *, \mathrm{p}<0.01 ; * * *, \mathrm{p}<0.001)$.

\section{Figure 2. Impact of HFD on elastin fragmentation in aorta and aortic pulse wave} velocity in $\mathbf{L D L R}^{-/-}$mice.

(A) Autofluorescence of aortic elastic fibers in normal diet (ND) and high fat diet (HFD) mice. White arrows indicate zone of elastin breaks. (B) Quantification of the number of breaks per elastic lamella in ND (white bar) and HFD (black bar) mice. Data represent the mean \pm SEM of 4 to 5 mice per group $(* * *, p<0.001)$. (C) Percent repartition of the number of breaks per elastic lamella in ND and HFD mice. The total circle represents $100 \%$. (D) Evolution of aPWV every 3 months in HFD mice. Data represent the mean \pm SEM of 7 to 9 mice. $(*, p<0.05$ compared to baseline).

\section{Figure 3. Effect of oseltamivir phosphate treatment on body weight and metabolic} parameters in HFD-fed $\mathrm{LDLR}^{-/-}$mice.

Plasma glucose (A), insulin (B), HOMA-IR index (C), and plasma total- (D), HDL- (E), and LDL- (F) cholesterol concetrations were measured from fasted LDLR $^{-/-}$mice fed with high fat diet (HFD) for 12 weeks, and treated (+OP) or not (-OP) with oseltamivir phosphate (50 
$\mathrm{mg} / \mathrm{kg}$, weekly). (G) Body weight. White bars (A-F) and white circles (G) correspond to vehicle-treated mice, and black bars (A-F) and black squares (G) correspond to OP-treated mice. Data represent the mean \pm SEM of 4 to 5 mice. (ns, non-significant; *, p<0.05).

Figure 4. Effect of oseltamivir phosphate treatment on elastin fragmentation in aorta and aortic pulse wave velocity in HFD-fed $\mathrm{LDLR}^{-/-}$mice.

(A) Quantification of the number of breaks per elastic lamella in mice treated with vehicle ($\mathrm{OP}$, white bar) or OP (+OP, black bar). Data represent the mean \pm SEM of 4 to 5 mice per group (**, $\mathrm{p}<0.01)$. (B) Percent repartition of the number of breaks per elastic lamella in mice treated with vehicle (-OP) or OP (+OP). The total circle represents 100\%. (C) Evolution of aPWV every 3 months in HFD mice treated with vehicle (-OP, white circles) or OP (+OP, black squares). Data represent the mean \pm SEM of 7 to 10 mice per group.

\section{Figure 5. Effect of oseltamivir phosphate treatment on atherosclerosis in aortic sinus} and carotid thrombosis in HFD-fed $\mathrm{LDLR}^{-/-}$mice.

(A) Quantification of atherosclerotic lesion sizes in the aortic roots of mice treated with vehicle (-OP) or OP (+OP). Left panel shows a representative photomicrograph of OROstained fatty streaks for each group (original magnification $\times 50$ ). Right panel shows quantitative analysis of atherosclerotic lesion sizes $\left(\mathrm{mm}^{2}\right)$ for mice treated with vehicle (-OP, white circles) and OP (+OP, black squares). The mean \pm SEM is shown. (B) Thrombotic occlusion time of mice treated with vehicle (-OP, white circles) or OP (+OP, black squares) after chemical injury of carotid arteries by $7.5 \% \mathrm{FeCl}_{3}$. The mean $\pm \mathrm{SEM}$ is shown. Top photographs show, as an illustration, a representative progression of thrombus formation in mice injected with vehicle (-OP) or OP (+OP). (C) Number of embols released during thrombus formation in the carotid arteries of mice treated with vehicle (-OP, white bar) and 
$\mathrm{OP}$ (+OP, black bar). Data represent the mean \pm SEM of 6 to 7 carotids per group. (ns, nonsignificant).

698

699

Figure 6. RT-qPCR analysis of gene expression in the liver of ND- and HFD-fed LDLR ${ }^{-/-}$ mice and impact of oseltamivir phosphate treatment.

(A) Liver weight of mice fed with normal diet (ND, white bar) or high fat diet (HFD) treated with vehicle (-OP, black bar) or OP (+OP, hatched black bar). Data represent the mean \pm SEM of 4 to 5 mice per group. (B, C) Total RNA was extracted from mice livers and analyzed by RT-qPCR as described in the Materials and Methods. Each gene expression was normalized using 2 reference genes (36B4 and RPS26) and results are presented as fold induction of ND mice. White bars, ND mice; black bars, HFD mice treated with vehicle; hatched black bars, HFD mice treated with OP. Data are expressed as mean \pm SEM of 4 to 5 mice per group. For each mouse, PCR reactions were performed in duplicate from 3 independent experiments. (ns, not significant; *, p<0.05; **, p<0.01; ***, p<0.001). 